\title{
Inequality in the Choice of Majors Among University Students of Different Economic Levels in the Context of the Expansion of Higher Education in China
}

\author{
Zishan Liu*
}

\author{
University College London, China \\ ${ }^{*}$ Corresponding author. Email: zishan.liu.21@ucl.ac.uk
}

\begin{abstract}
The expansion of higher education in China has increased access to a large number of universities, but it has brought inequality in the choice of majors for university students. To examine the relationship between the choice of majors of Chinese higher education students and the level of economic development of their regions, which has been rapidly expanding since 1999, this study analyses national economic development and national higher education university enrolment data collected by China's National Bureau of Statistics over the decade 2010-2019. This study classifies high, medium and low-income professions and regions with high, medium and low levels of economic development based on the per capita income of graduates in each profession and the per capita GDP of each region in China respectively. The results show that the economic level of a student's region affects the student's major choice, with a higher proportion of students in high-economic level regions choosing high-income majors, while a higher proportion of students in low-income regions choose low-income majors. This gap has been increasing over the past decade.
\end{abstract}

Keywords: Higher education expansion, Major selection, Regional economical levels.

\section{INTRODUCTION}

Since 1999, the Chinese government required higher education institutions to implement enrollment expansion policies and other related measures to ensure that more people have access to higher education opportunities. Since the implementation of the policy of expansion of higher education, there is a positive effect that more than $83 \%$ of high school students have been able to enter higher education in only four years, compared with only 46\% in 1998 (Li, 2010). From then on, China's higher education has gradually shifted from the elite education stage to the popularization; it allows families and disadvantaged groups in the lower social strata to gain more educational opportunities.

However, several pieces of research suggested the determinants of demand for higher education and had documented the trend of unequal access to college in China (Wu and Zhang 2010; Li et al. 2014). The proportion of students from economically disadvantaged areas and low-income families in Chinese higher education has not increased (Li, 2010). By analyzing the Chinese college enrollment data, Li (2010) found that covered by the increasing number of enrollment, the enrollment ratio of urban and rural students even had more disparity.

Yet the existing research on the inequalities that arise in the expansion of higher education in China has mainly been observed and demonstrated through enrolment rates; what has received little attention is the inequalities encountered by university freshmen when choosing a major.

This article examines for the first time the inequalities and differences in the choice of majors of students in regions with different levels of economic development in the context of the expansion of higher education in China. Focusing on the changes in the trends of Chinese higher education students' choice of majors over the past decade from a macro perspective, I pose the following two research questions:

1. Are there differences in students' choice of profession among regions with different levels of economic development?

2. How do trends in students' choice of major vary among regions with low, medium and high levels of economic development? 


\section{INTRODUCTION TO THE CHINESE COLLEGE ENTRANCE EXAMINATION SYSTEM}

To be accepted into university, high school graduates in the China region are required to take a competitive, nationally-organised college entrance examination. The examination results of the Gao Kao will determine the students' access to universities, and students will fill in the universities and majors they wish to apply to in their application forms, after Gao Kao. Each university has its minimum mark, based on a nationally required quota, and applicants can access to the university if they exceed its minimum mark.

Usually, places at higher education institutions are allocated in equal proportions according to the population of each region. There is no limit to the number of schools and subjects that a student can apply to, and the university will then decide whether to accept the student based on his or her GCE scores. It is worth noting that students must take the entrance examination in their place of residence, which results in most entrance examination candidates taking the local entrance examination in their place of birth or permanent residence.This means that most students will be educated in their domicile and that students from different regions can each be seen as a collective, so it is possible to assess the tendency of students in this region to choose their major by collecting data from each region.

\section{LITERATURE REVIEW}

Regarding the relationship between higher education expansion and equality of educational opportunity, sociological and economic studies have provided many competing hypotheses and inconsistent empirical evidence.

Some scholars (Raftery and Hout, 1993; Shavit and Blossfeld, 1993) have suggested that higher education expansion does not reduce inequality in education. A well-known hypothesis is maximally maintained inequality (MMI), which argue that inequality in educational attainment between any two social groups will persist until the advantaged class reaches a point that nearly all of its members attain the desired level of education (a saturation point at a given level of education). Another well-known hypothesis is the EMI theory. That is, even if the saturation point of higher education is to be reached, inequality of opportunity is likely to persist if the advantaged groups receive disproportionate access to the most selective universities (Lucas, 2001). Since students from better-off and higher socially advantaged families are more likely to have access to higher education, in this situation, the expansion of education in China will not improve equity of access to higher education. Indeed, studies have shown that, as in other countries, education expansion in China has not led to equal distribution of educational opportunities, and the least socioeconomically advantaged students are missing out $(\mathrm{Ou}$ and $\mathrm{Hou}$, 2019).

A new perspective put forward by Marios (2020) is that in the background of mass opportunities to attend college, the inequality of major selection might be a new issue we do not notice before. Taking a private university in Cyprus, his research results do not support that the expansion of education in Cyprus has eliminated educational inequality. He found that in the university prestigious majors offer very limited places (such as medicine), and tuition fees are even higher. In reality, these majors can only become the choice of wealthy families.

The same thing happened in Chinese higher education, Chinese students have been provided mass opportunities to enter colleges and universities, a new style of education inequality is noticed recently, the gap of major selection. A considerable number of rural students and students from lower-class families meet a limitation of major selection, not because of the institute policy, but for their family economic level and the limitations of the eyesight due to the living environment (Fan, J., 2009). In the case study in Liaoning Province, China, the researcher noted that gender, high school level, and social employment environment were important considerations for students when choosing a major (Yunan, Y., 2011).

Although there are subsidised scholarships at universities to help needy students, very few students benefit from them (Yan Liu, 2006). As the increase in the number of students in higher education since the expansion of education has increased the financial pressure on universities, the current scholarships for higher education in China are mainly intended to reward students for outstanding academic performance and achievement (R. Zhao, 2011).

From the existing studies on the expansion of higher education in China, the main focus has been on equity in educational opportunities to enter university, with less research focusing on students' professional choices. The research has been conducted by testing the MMI and EMI hypotheses, and in regression analysis of data such as national higher education enrolment, comparative analysis of higher education expansion between different regions is lacking. Also, in terms of studies on major choice, few extant studies focus on changes in major choice trends resulting from higher education expansion.

\section{METHODOLOGY}

The research used a quantitative approach to analyse the major selection data in a Chinese university in the 
last decade (2010-2019). The population considered for this study is defined as the students who were enrolled by a Chinese public university. The data analyzed for this study were obtained from the National Bureau of Statistics of China and the China Education Yearbook 2010-2019, including the number of graduates of various majors in the country each year and the number of undergraduate graduates of the total national universities each year.

To find the major selection trend of different backgrounds students, the first step will be classification. Divide students into several grades according to the degree of economic development of their province. The classification of the level of economic development is based on the ranking of the region's GDP per capita among the 31 regions in China. It also ranks the income of majors based on the average salary of graduates of each major, classifying lowincome, middle-income and high-income majors, based on the ranking of the average income of the major among all major categories of higher education in China. Then, using backgrounds' grades and the years as analysis elements, the statistic chart can demonstrate the changing trend of students' major choices from different backgrounds.

In addition to the change in the absolute number of graduates of different backgrounds and majors each year, the relative proportions of different classes of students' professional choices should also be analyzed to determine whether the educational inequality in professional choices has improved since the implementation of the educational expansion policy.

\section{RESULTS}

According to the official data published by the National Bureau of Statistics of China for 2018-2020 as shown in Figure 1, it can be seen that the two municipalities of Beijing and Shanghai have the highest GDP per capita, both exceeding 140,000 yuan. Among the 31 provinces in the country, Anhui and Hunan have a GDP per capita of around RMB 60,000, which is in the middle of the country. The two most underdeveloped provinces are Heilongjiang and Gansu, with a GDP per capita of no more than RMB 40,000. Therefore, the six regions mentioned above, representing regions with high, medium and low economic levels in China, were chosen for this study to analyse the proportion of students enrolled in different majors in higher education in these regions, to investigate whether the level of regional economic development affects the willingness of local students to choose their majors.

Next, according to the data published by the National Bureau of Statistics of China on the average annual wages of workers in various professions in 2018-
2019, as shown in Figure 2 below, "information technology" and "finance" were selected to represent high-income professions, "medicine" and "public administration" to represent middle-income professions, and "agriculture" and "forestry" to represent low-income professions.



Figure 1 GDP per capita by province (Official Chinese figures for 2018-2020).

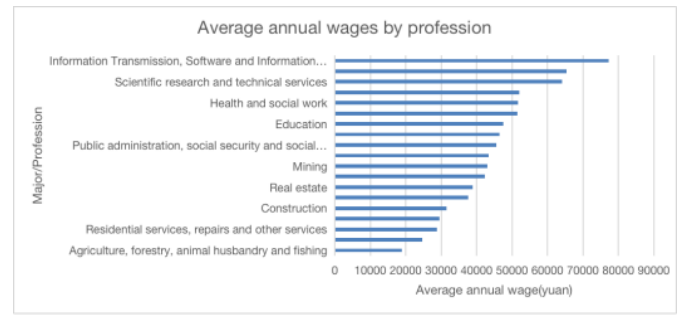

Figure 2 Average annual wages by profession (20182019)

To determine the enrolment of university majors in regions with different levels of economic development (low, medium and high emergency levels), the study retrieved official data from the Chinese Ministry of Education and the Chinese Bureau of Statistics to obtain twenty years of historical undergraduate enrolment data and the number of students enrolled in different majors in each province in the last ten years of the Chinese Education Yearbook. 


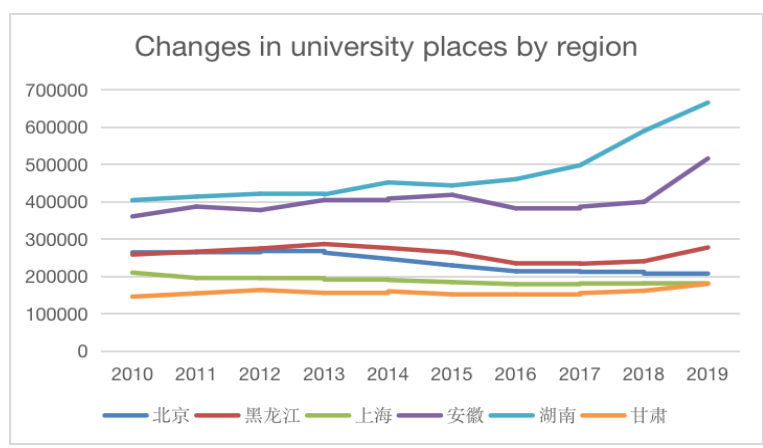

Figure 3 Changes in university places of six regions in China

Figure 3 shows the number of university enrolments in the six provinces surveyed over the decade 20102019. The trend shows that only two provinces with a medium level of economic development, Anhui and Hunan, have seen a relatively significant increase in enrolments over the decade, while the other four provinces have remained largely stable.

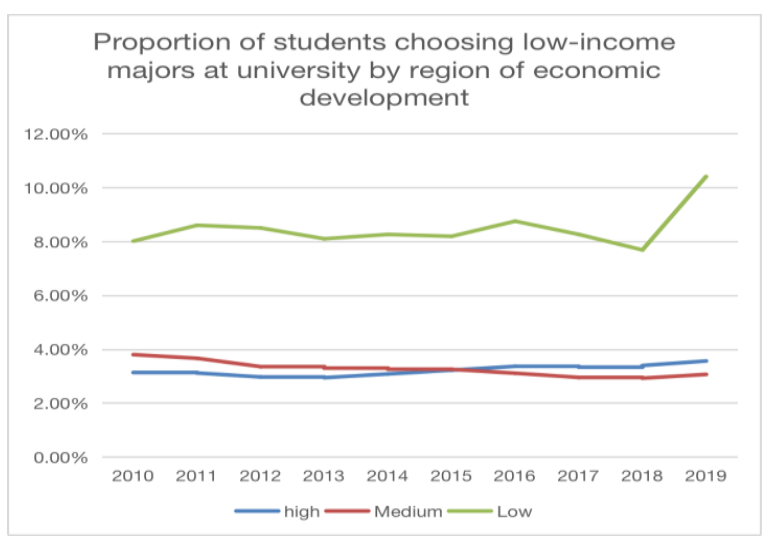

Figure 4 Proportion of students choosing low-income majors at university by regions of economic development

Figure 4 shows the choice of undergraduate majors for the low-income majors "Agriculture" and "Forestry" in regions with different levels of economic development. The percentages in Figure 4 represent the proportion of students in the province who chose 'Agriculture' and 'Forestry' out of all students admitted to universities in that year. The three different coloured lines represent high-income provinces, medium economic development provinces and low economic development provinces. It can be seen that the trend for all three categories is relatively stable over the decade 2010-2019, with the number of students in middle and high-income areas choosing agronomy and forestry remaining between $2 \%$ and $3 \%$, while the number of students in low-income areas choosing these two majors is higher, at over $8 \%$, and has increased significantly over $10 \%$ in $2018-2019$.

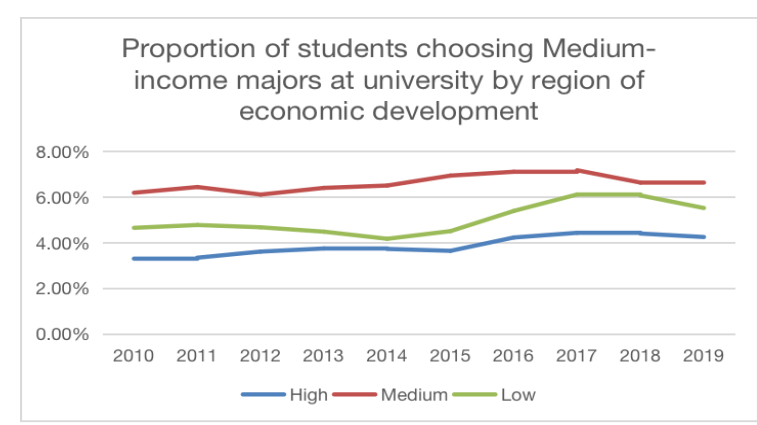

Figure 5 Proportion of students choosing Mediumincome majors at university by region of economic development

Figure 5 shows the enrolment in the middle-income disciplines of Medicine and Public Administration. It can be seen that the smallest proportion of students from high-income areas choose these two majors, while they are more popular in middle-income areas, where they have remained above $6 \%$ since 2010 .

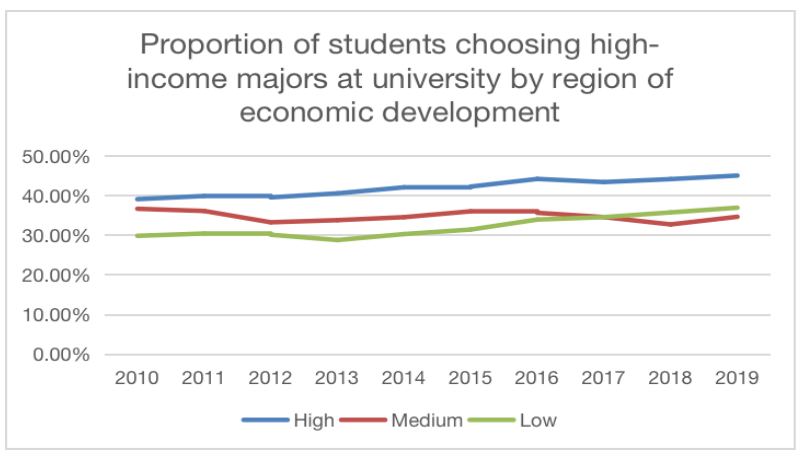

Figure 6 Proportion of students choosing high-income majors at university by region of economic development

Shown in figure 6 , the proportion of students choosing the high-income majors "Information Technology" and "Finance" in different regions of economic development, and it can be seen that the proportion of students in different regions has not changed significantly over the decade 2010-2019. In terms of overall trends, the proportion of students choosing high-income majors has been slowly increasing over the decade, but compared to 2010 when $39 \%$ of students in high-income, $36 \%$ in middle-income and $29 \%$ in low-income regions were able to choose electronics and finance as their majors, the gap between the three regions has increased to $45 \%$ in high-income regions and $37 \%$ and $34 \%$ in middle- and low-income regions respectively in 2019 .

\section{DISCUSSION}

The basic conclusions that can be drawn from the data collected on the number of university admissions and professional admissions in the six provinces of Beijing, Shanghai, Anhui, Hunan, Heilongjiang and Gansu over the last ten years are 1)Access to tertiary 
education has increased significantly in the decade 2010-2019 in the middle-income regions, while the number of places in the higher and lower-income regions has remained stable.2)Students in different economic regions have their own preferences when choosing their majors, with a higher proportion of students in low-income regions choosing low-income majors and a higher proportion of students in highincome regions choosing high-income majors.

First, there is geographical variability in the expansion of higher education places over the decade 2010-2019, and for the six provinces covered in this study, the two provinces with significant increases in enrolment are both provinces with medium levels of economic development. This may be related to the prioritization of higher education development in China mentioned in other studies, i.e. since the 1998 reform of higher education expansion, higher education in regions with high levels of economic development was implemented first and then gradually to other regions. This may have resulted in economically developed regions having completed their place expansion in the first decade of reform, while medium economic development regions were in the process of increasing university places in the decade 2010-2019.

Secondly, the research data suggest that the choice of university students' majors does correlate to a certain extent with the level of economic development of the province in which the students live. This is particularly evident in the low economic development regions of Heilongjiang and Gansu, where the proportion of students choosing low-income majors is significantly higher, almost twice as high as in the middle and high economic development regions. The strong link between regional economic and major choices is also evident in the proportion of middle- and high-income major choices - a higher proportion of students in middleincome regions choose middle-income majors, and a higher proportion of students in high-income regions choose high-income majors.

If we correlate the current major industries in the student's region with the data collected in this study on major choice, we may find that there is an inevitable influence of the regional economy on students' major choices. Based on data from the China Statistical Yearbook, it was found that the main pillar industries of the two provinces in this study, Heilongjiang and Gansu, which represent low-income regions, are agriculture, forestry and animal husbandry, and that local jobs are more in demand Jobs are also more in demand in "low-income" professions. Likewise, jobs for middle and high-income majors are more likely to be located in areas with medium to high levels of economic development. Thus, students in different economic development areas are exposed to different occupations daily before college, and they see different perspectives on job demand, which is likely to be a factor in the major choice.

Another conclusion suggested by the research data is that the differences in students' choice of majors between regions with different levels of economic development have not been eroded by the current reforms of educational expansion implemented in the decade 2010-2019, and even the differences in the choice of majors between regions have widened. From the perspective of educational equity in major choice, the educational expansion reform did not achieve its aim of reducing the differentiation between regions to promote educational equity.

However, as this study mainly uses national historical statistics as a data source for macro perspective analysis, it can only roughly reflect the current situation of university students' major choices through the several provinces surveyed, and it is difficult to find more individualized influencing factors. In the future, it is necessary to select specific schools in specific regions and conduct more microscopic and indepth questionnaires and interview studies to determine the specific factors influencing students' choice of majors.

\section{CONCLUSION}

In many studies of China's educational expansion, the first observed is often the direct change of educational opportunities, but I think it is very necessary to study the impact of educational expansion from a more dynamic perspective.

From the perspective of major choice, this study found that students in different regions of China have a clear preference in choosing their majors. This preference is closely related to the economic level and industrial structure of the region, for example, the main economic sectors in low-income regions are forestry and agriculture, which makes local students more likely to choose agriculture and forestry majors than in other regions. Similarly, finance majors are more popular in areas with high levels of economic development.

In addition, the study shows that the abovementioned phenomenon has not weakened but has increased over the last 10 years of higher education expansion. The fact that the proportion of students choosing low-income majors has increased in low economic development areas, while the proportion of students choosing high-income majors has increased faster in high economic development areas, suggests that regional economic inequalities in the choice of higher education majors may be increasing.

This means that the expansion of higher education in China needs to focus not only on increasing access but also on a new kind of educational inequality-the 
difference in choice of profession due to uneven regional economic development.

For small tables, please place it within a column and bigger table be placed in a text frame spanning to both columns. Use the Table facility available within the MSWord. The font in the row header should be bold and you can use the style available from the style palette.

\section{REFERENCES}

[1] Li, c. (2010). Higher Education Expansion and Inequality of Educational Opportunity - An Examination of the Equalizing Effect of Higher Education Expansion. Sociological Research. (03), $82-113+244$ doi:10.19934/j.cnki.shxyj.2010.03.004.

[2] Marios \& Zheng You. (2020).Unintended Consequences of Mass Participation in Higher Education: New Forms of Social Inequality and Brain Drain. Journal of Education (06), 71-77. doi:10.14082/j.cnki.1673-1298.2020.06.008.

[3] Li, S., Whalley, J., \& Xing, C. (2014). China's higher education expansion and unemployment of college graduates. China Economic Review, 30, 567-582. DOI: 10.1016/j.chieco.2013.08.002

[4] Wu, X. and Zhang, Z. (2010), "Changes in educational inequality in China, 1990-2005: Evidence from the population census data", Hannum, E., Park, H. and Goto Butler, Y. (Ed.) Globalization, Changing Demographics, and Educational Challenges in East Asia (Research in the Sociology of Education, Vol. 17), Emerald Group Publishing Limited, Bingley, pp. 123-152. https://doi-org.libproxy.ucl.ac.uk/10.1108/S14793539(2010)0000017007
[5] Mingcheng, F. (2009). A study on the choice of majors in Chinese universities (Doctoral dissertation, Xiamen University). https://kns.cnki.net/KCMS/detail/detail.aspx?dbna $\mathrm{me}=\mathrm{CDFD} 0911 \&$ filename $=2009208557 . \mathrm{nh}$

[6] Ou, D., Hou, Y. Bigger Pie, Bigger Slice? The Impact of Higher Education Expansion on Educational Opportunity in China. Res High Educ 60, 358-391 https://doi.org/10.1007/s11162-018-9514-2

(2019).

[7] Yunan, Y. A study on the factors influencing the choice of major in college entrance examination [D]. Liaoning Normal University, 2011.

[8] Yan Liu. Study on Scholarship in Chinese Universities [D]. Huazhong University of Science and Technology, 2006.

[9] Raftery, A. E., \& Hout, M. (1993). Maximally Maintained Inequality: Expansion, Reform, and Opportunity in Irish Education, 1921-75. Sociology of Education, 66(1), 41-62. https://doi.org/10.2307/2112784

[10] R. Zhao (2011). An analysis of present-day domestic undergraduate university scholarship system based on decision theory [J]. Eastern Enterprise Culture, 2011(18):283-285.

[11] Shavit, Y., \& Blossfeld, H. P. (1993). Persistent Inequality: Changing Educational Attainment in Thirteen Countries. Social Inequality Series. Westview Press, 5500 Central Avenue, Boulder, CO 80301-2847. 